\title{
Journal of Communications
}

\section{CONTENTS}

\section{Volume 9, Number 2, February 2014}

Enhanced Performance of SAC-OCDMA System based on SPD Detection Utilizing EDFA for Access Networks.... 99 Sarah G. Abdulqader, Syed A. Aljunid, Hamza M. R. Al-khafaji, and Hilal A. Fadhi

Energy Efficient CCC based AODV Routing Protocol for Cognitive Radio Ad-Hoc Networks 107

Satish Anamalamudi, Chang Liu, and Minglu Jin

Precoded Spatial Multiplexing MIMO Systems in Time-Varying Fading Channel.....

Abdullatif S. Kharwat

Performance of Star 16/64QAM Schemes Using Turbo FDE with Iterative Decision-Directed Channel Estimation for DFT-Precoded OFDMA

Chihiro Mori, Mamoru Sawahash, Teruo Kawamura, and Nobuhiko Miki

Study on Denial of Service against Underwater Acoustic Networks

Yangze Dong, Hefeng Dong, and Gangqiang Zhang

CLAM: Cross-layer Localized Authentication Mechanism based on Proxy MIPv6 and SIP in Next Generation Networks

Muhammad Zubair, Xiangwei Kong, and Saeed Mahfooz

A Precoding Method for Multiple Antenna System on the Riemannian Manifold

Lin Zhang and S. H. Leung

Decentralized Cooperative Communication Framework for Heterogeneous Multi Agent System

Herdawatie Abdul Kadir and Mohd Rizal Arshad

Single Value SRS (SV-SRS) Feedback Control Method for LTE Uplink Transmission

N. A. Kaim Khani, Z. Chen, and F. Yin

Self-Adjustment Mechanism Guaranteeing Asymptotic Stability of Clusters Formed by Autonomous Decentralized Mechanism 180

Hiroki Takayama, Sota Hatakeyama, and Masaki Aida

Guard Time Optimization for Capacity Maximization of BPSK Impulse UWB Communications 188 Abdallah Hamini, Jean-Yves Baudais, Andrea M. Tonello, and Jean-François Hélard 